\title{
Liliana Curcio Experiences in a Modeling Laboratory: and Roberto Didactic Exhibit curated by the Istituto Statale Di Martino d'Arte di Monza in collaboration with the Liceo Artistico di Busto Arsizio
}

The model-building laboratory, more than just a place for building models, is a place of study, research, thought processes, and ideation; the place where the acquisition and transmission of knowledge constitutes a fundamental method for growth both for the students as well as the instructors; the place where disciplines are not always considered akin can meet and be compared. Architecture and mathematics come together in this exhibit for Nexus 2000 at the Museum of Architecture (MusArc) in Ferrara, Italy.

\section{Introduction}

One of the strongest objectives invigorating the present experiment, still in progress today at the Istituto Statale d'Arte (ISA) of Monza, is that of considering different ways of knowing different kinds of languages, i.e., the language of logic, the expressive-communicative language, and the culture of design. In this context, the various disciplinary fields can be considered as areas making use of specific instruments for investigating the world, each characterised by its own unique approach. The language of logic is seconded by the mathematical disciplines, by the theory and applications of descriptive and projective geometry, and by workshops or laboratories for applied arts, disciplines apparently diverse but united in an extraordinary way by common, invisible, threads that interweave at the boundaries of each discipline.

In the exhibit mounted at the MusArc, National Museum of Architecture, in Ferrara, Italy, on occasion of the Nexus 2000 conference on architecture and mathematics, the results of some of the didactic curricula followed within the context of the language of logic were displayed, in particular those of the "Modeling Laboratory". This laboratory, more than just a place for building models, is a place of study, research, thought processes, and ideation; the place where the acquisition and transmission of knowledge constitutes a fundamental method for growth both for the students as well as the instructors; the place where disciplines are not always considered akin can meet and be compared. Here the model is seen not only as a completed whole, but in its various facets:

- as representation and therefore as close to the object; this is the field of reproduction; 
- as description that links forms and concepts; this is the field of experimentation;

- as a fundamentally close-up interpretation; this is the field of design.

The primary object of study and research has been to investigate form. It is, in fact, the approach specific to the study of form that links the disciplines of the language of logic: knowing, classifying, distinguishing, inventing families of forms on the basis of mathematical properties that act as morphogenetic agents. The forms are then analysed and studied in relation to their reciprocal functions, their stability, to their possible modelling; each disciplinary language, in its own specific ways, retraces this same process, while at the same time requiring the support of other disciplines and illuminating how their processes are related. Thus the model is understood in its most complete sense, and describes totally the process of study and research that each discipline goes through in the diverse phases of its interpretation, its description and its realisation. The model becomes experimental, heuristic, sometimes simulating situations that allow a glimpse of itineraries that are new but that, in their newness, are still obscure; it is not always easy to identify the surest pathway for investigation.

The didactic processes analysed in the exhibit constitute examples of the relationships between mathematics and architecture and the connections between these and other disciplines such as physics, chemistry, science, history of architecture and design. The multimedial nature of the investigations guarantee a final product that needs no translating, interpretation or intermediaries but that carries in itself a message that can be read in all languages.

\section{The Materials Exhibited}

The materials in the exhibit were of a heterogenous nature, and though not always the fruit of the teaching curriculum, they are in any case "products" of the school's cultural climate. An example of this is the great tetrahelical tower built by two professors as an extracurricular project. Other projects represent moments of reflection on pure geometric forms, though attention is always paid to their linguistic merit and to the opportunities they offer for this project.

In the room of the exhibit dedicated to polyhedrons (Figure 1) was a series of plates of text produced by the fourth class of the fine arts section of the Liceo Artistico of Busto Arsizio, in which the problem of classifying the families of polyhedrons was examined with rigor and clarity. In the same section were found a number of models of polyhedra that dealt with the problem of conjungation between these ideal forms and their relationship to different materials and constructive techniques. At the end of the section dealing with "pure" polyhedrons were found the polytopes of the fourth dimension; the famous hypercube was not represented, but in compensation there was an previously un-exhibited model of the 120-cell (!), which, with its fascinating knotting, brings home the difficulty of representing such complexity.

Also represented in the exhibit was the work of students who graduated some time ago from the school, but in the work of whom the spirit of the school's work is easily recognisable. The first of these was a project on the tetrahedron carried out in a series of 
commentated and annotated plates that make clear the richness to be found in these forms, understood in the largest sense of the term. The other project concerned the field of lightweight construction, with an emphasis on the technological aspect of the problem. The object represented was a universal joint for a two-layer geodetic dome capable of handling twelve members (Figure 2). These two projects each represented a richness of conception and visual thinking.

Other projects exhibited represented, through models, reflections and analyses of the works of Ledoux (Figure 3) and Le Corbusier (Figure 4), great architects who made use of the esthetics and the rationality of geometry. This is only a limited representation of a tradition in the school that has seen studies undertaken on the works of architect-engineers such as Nervi, Musmeci, Torroja, Candela, Frei Otto, Le Ricolais, the influence of whom was felt in the projects on display. In particular there was a reference to the work of Fuller: in the tetrahelix; in the great "geoscopic" sphere (Figure 5); in references to the geoid; and more

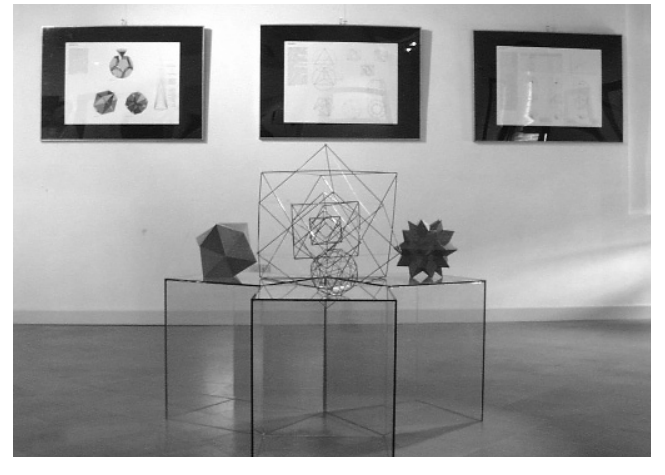

Figure 1

MusArc, Museum of Architecture, Ferrara, Italy. The room of the exhibit dedicated to polyhedrons.

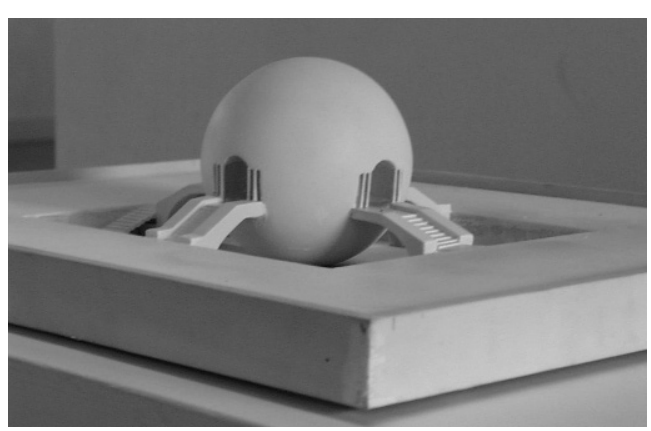

Figure 3

Model of the house of a gardener by utopian architect Ledoux.

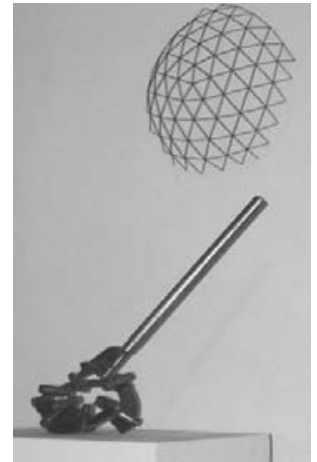

Figure 2

A universal joint for a two-layer geodetic dome capable of handling twelve members.

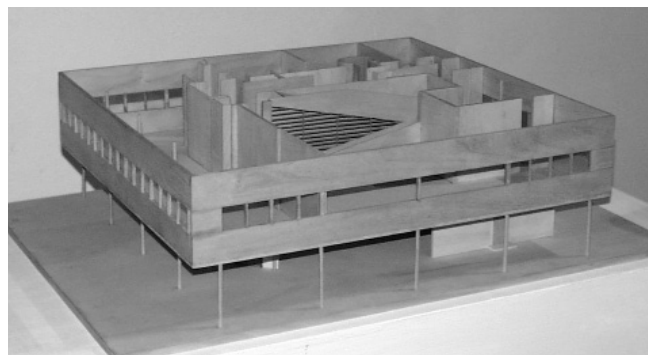

Figure 4

Model of the Villa Savoye by Le Corbusier. 
generally, in the interdisciplinary approach to the study of form. There were a series of plates and models dedicated to the theme of tiling the plane. The abstract geometrical problem was represented, for example, by the box of polygonal tiles made of different woods, in which even a simple arrangement of the forms becomes a game of colors, thicknesses, finishes and reflections that has it own tactile attraction.

Other objects in the exhibit did not fall into any precise category but in any case reflected the spirit and intention that formed them. Their presence, justified in any case by the beauty of their forms, indicated new, possible courses of investigation.

It is evident that the search for harmony and perfect belong to the patrimony of different disciplines and it is important that study and research aim to unite these curricula. Such an aim may be seen in poetry and in the thoughts of scholars, architects and artists. We cite two such figures - far apart in time but very close in terms of their aims. The first, Leon Battista Alberti, said that "Beauty [is a] harmony of all the parts, in whatsoever subject is appears, fitted together with such proportion and connection, that nothing could be added, diminshed or altered, but for the worse." On the other hand, for Alberti, numerus was number as quantity, number as quality, and number as form. Number and form are closely interconnected; in fact, the Latin term numerus, from which Alberti took his inspiration, had a double significance, both as number (arithmos) and as form (rhythmos). The same term could be translated as either, "rhythmos and arithmos were associated by dint of their both being delimiting factors. Rhythmos delimited the infinite with the aim of producing form, arithmos delimited the infinite with the aim of producing a specific quantity." The second figure that we cite is Le Corbusier, who, in chapter three of Le Modulor asserts:

Mathematics are the masterly edifices conceived by man for understanding the universe...Architecture is not a phenomenon in synchrony, but successive, made of spectacles that join to one another and are successive in time and space, just as in music... The reigning harmony in all things, regulating the things around our lives,

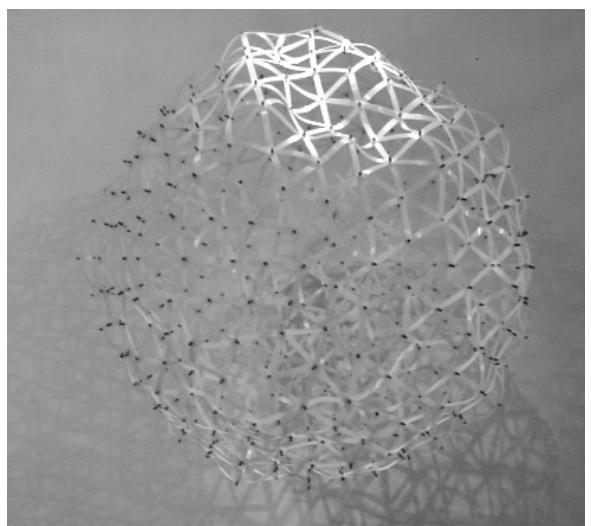

Figure 5

Model of the "geoscopic sphere".

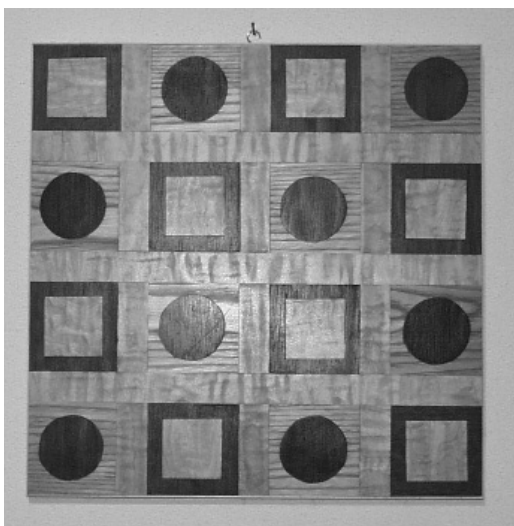

Figure 6

The pavement pattern from the Pantheon, Rome, executed in wood intarsia (marquetry). 


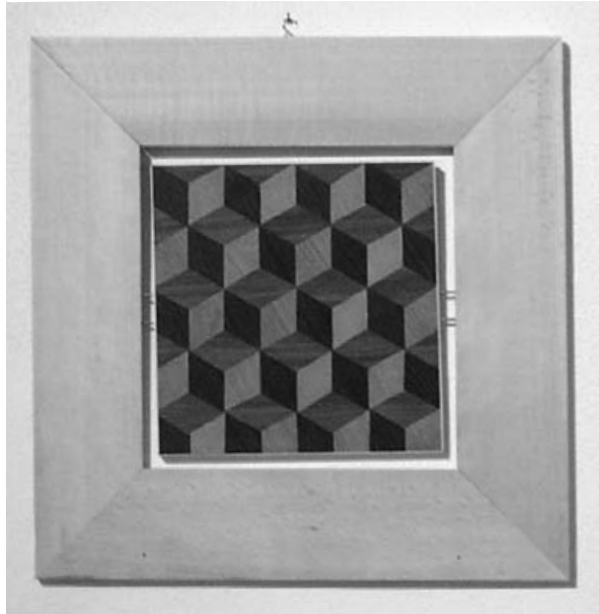

Figure. 7

The pavement pattern from the Cathedral of Lucca (stepped cubes), executed in wood intarsia (marquetry).

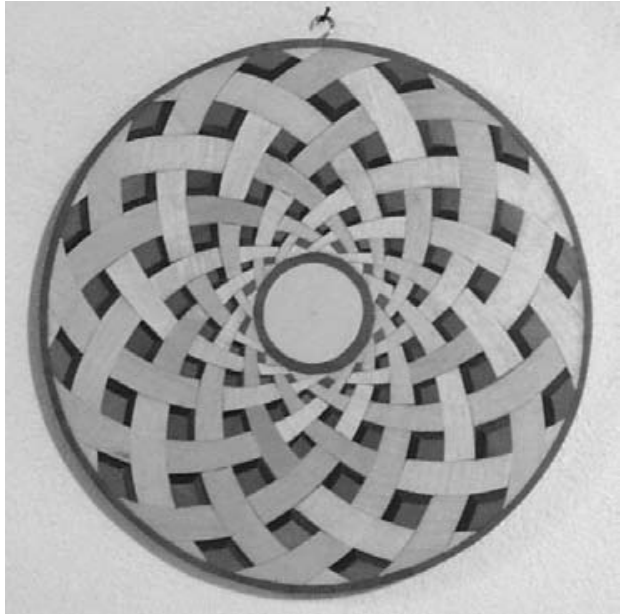

Figure 8

A pavement pattern from the Cathedral of Florence, executed in wood intarsia (marquetry).

is the spontaneous, assiduous and irrenounceable exhaling of man who has been inhaled to by a force, the divine force, charged with a mission: to find paradise on earth...

The spirit on which the Modelling Laboratory is based is especially inspired by these two ways of thinking. It is very important to remember that beauty and harmony are not necessarily restricted to one disciplinary environment but are rather part of the culture and labor of all disciplines; it is through the convergence in the same direction them all - each with its own specificity - that each is able to manifest beauty and harmony through different forms and typologies.

\section{Tilings of the Plane and Italian Pavements}

This section of the exhibit presents a different didactic curriculum, undertaken by students of the class $1 \mathrm{~B}$ within the Laboratory of the Language of Logic. In this laboratory, the disciplines of mathematics and descriptive geometry converge with the craft of cabinet-making. The curriculum involved an analysis of geometric transformations in the plane from a mathematic point of view and the synthetic treatment of the same, analysed through sketches and plates, with the aim of being able to recognize the applications and combinations of plane transformations in architecture. Part of the coursework was a video on the mosaics of the Alhambra in Granada, in which, as is by now well-known, all seventeen groups of symmetries were found and catalogued by Spanish mathematician Montesinos. It was particularly significant for the students to reproduce some examples in architecture, especially the execution of some models in wood intarsia or marquetry of noted Italian paving patterns (Figure 6, Figure 7, Figure 8) chosen in collaboration with architect Kim Williams, author of the book Italian Pavements: 
Patterns in Space. The works produced by these first-year students make evident the didactic value of this exercise. From an operative point of view the first steps were to produce the regular and semi-regular tilings of the plane, which were constructed of "straws". In a later moment some students built the "tilings box" using different kinds of wood. From the images of pavement patterns, the students were required to produce on their own geometric structures of every kind of pavement; only in the more complex were they assisted by their professors. Finally, to this was added some reproductions of pavements through computerisations using Cabri II software. What was presented was a work in progress. Some of the models were not yet complete, but all were particularly meaningful in terms of demonstrating the difficulties encountered, the analyses undertaken and the resolutive forms chosen.

In appendix to this work were some plates of analyses of Islamic tilings taken from the classic text by Burgoing, which were produced by some students of the fifth-year class, testifying to the tranversality and richness of this themes, which can be dealt didactically more than once and in various ways. Also on display was a group of polyhedra, many of which were taken from the drawings by Leonardo da Vinci for Luca Pacioli's De divina proportione (Figure 9). A curious "flower" in the middle of the room visually recalled some of the rosette pavement designs on display, even though in reality it was a model of a collapsed hyperboloid that produced this effect.

\section{The Tetrabelix}

The tower placed in the center of the main space of the exhibit represented the tetrahelix (Figure 10), a geometric figure not very well-known but visually fascinating. The form is composed by a theoretically infinite column of regular tetrahedra, joined in such a way so that each polyhedron has one face in common with two other polyhedra, and shares a vertex with three others. All the vertices of the structure are equal, and there are six corners in every vertex.

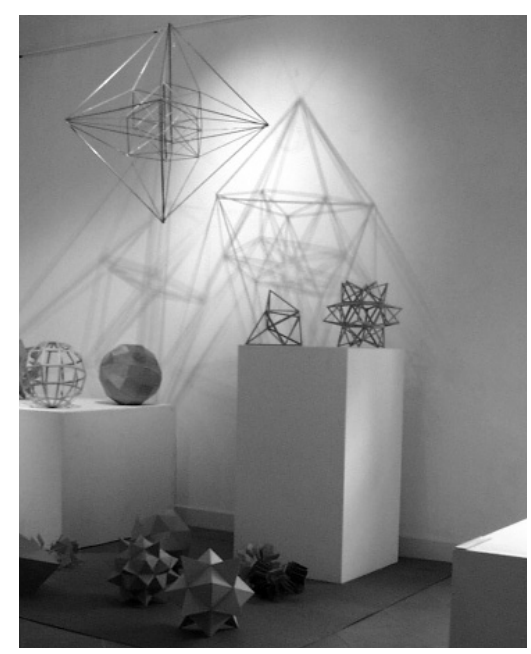

Figure 9

A group of polyhedra, many of which were taken from the drawings by Leonardo da Vinci for Luca Pacioli's De divina proportione. 
The tetrahelix was studied for the first time in the 1950's by Buckminster Fuller (18951983), one of the major exponents of the American twentieth century, active as an architect, engineer, philosopher and student of nature, from which, with an objective eye and the breadth of a great utopian, he drew principles and metaphors for his projects for the whole of humanity. For Fuller, the tetrahelix was not only a well-known regular solid, but also represented the "spatial quantum", that is, the prime element of three-dimensional space; the four vertices of the tetrahelix are the minimum number of points with which it is possible to define a figure in space. Some images from Synergetics. Exploration in the Geometry of Thinking (1975) are helpful in understanding the determining role palyed by the tetrahelical metaphor in Fuller's thinking: a physical model that helps to understand material state and behaviour, but also a linguistic structure that indicates the ways of conjugating prime entities in order to form multiplicities.

The tetrahelix is seen as a linear structure of maximum theoretical rigidity and as a physical model - conceptually, the double helix of DNA. Fuller proposed analogies between the behaviour of the four GTCA bases (four, as in the vertices of the "spatial quantum") and the most complex tetrahelical structure that joins its own elements according to an analogous helicoidal pattern.

The helicoidal model visualises in an immediate way the helixes that are "nested" in the tetrahelix. The model is obtained through an ideal projection of the figure on a cylinder in which it is inscribed, starting from all the points of its axis. The broken corners are transformed into continuous helixes that are easy to read. Helixes of the same type, that is, superimposed on each other with a rotation, have the same color. It is easily observed that the whole tower, theoretically to the infinite, is composed of only six helixes of three different types:

- The white helix passes through all vertices;

- The two green helixes skip alternate vertices;

- The three red helixes skip every third vertex.

The black helix and the three red ones go in the same direction; the two green helixes go in opposite directions; if the green ones are clockwise (dextrarotatory), the others are counterclockwise (laevorotatory) and vice versa.

These patterns evoke other images: phyllotaxis, some helical reinforcing structures present in marine animals of tubular form or in linear structures designed by man.

First published in the NNJ online July 2000

\section{About the Istituto statale d'art sperimentale (ISA Monza)}

The Istituto statale d'arte sperimentale of Monza has been an experimental school in the fields of design, visual communication, industrial design and environmental design for twenty years. The experimentation aims to furnish students with both pre-professional competence as well as critical instruments for understanding in terms of design culture and visual linguistics. The study cycle lasts five years and concludes with a diploma (the Italian 
maturità). The ISA Monza is the only such school in the province of Milan. The school is housed in a wing of the Villa Reale, which was the home for the Istituto per le industrie artistiche (Institute for Artistic Industries) in the 1930's. This was connected with the Milan and Monza biennale and triennale exhibits, and thus with the birth of modern design and architecture in Italy. The ISA Monza features studios in sculpture, photography, cinema, television, graphic techniques, model building, cabinet making and metal working. It also offers classes in the history of art, chemistry and technology, physics, science and English. 